\title{
Impact of Pilot Design on Achievable Data Rates in Multiple Antenna Multiuser TDD Systems *
}

\author{
Dragan Samardzija \\ Bell Laboratories, Alcatel Lucent \\ 791 Holmdel-Keyport Road \\ Holmdel, NJ 07733, USA \\ dragan@bell-labs.com
}

\author{
Narayan Mandayam \\ WINLAB, Rutgers University \\ 671 Route 1 South \\ North Brunswick, NJ 08902, USA \\ narayan@winlab.rutgers.edu
}

\begin{abstract}
In this paper we study the effects of practical pilot-assisted channel state estimation on the achievable information theoretic data rates (uplink and downlink) in a multiple antenna multiuser TDD system. Specifically, we consider a wireless system with multiple antennas at the base station and a number of mobile terminals each with a single antenna. We analyze the performance of uplink multiuser detection and downlink transmitter optimization that are based on linear spatial filtering. Using a discrete version of the continuously time-varying wireless channel we analyze how a lower bound on the achievable data rates depends on (1) the arrangement of pilot symbols (preamble or postamble); (2) the duration of the uplink and downlink transmissions; and (3) the percentage power allocated to the pilot. Furthermore, we also present the effects of the terminal speeds on the achievable data rates, thereby providing prescriptions for the practical design of the pilot. Specifically, our results point to an uplink postamble with flexible percentage (about 20 - $25 \%$ ) pilot power allocation, and transmission durations tailored for specific ranges of terminal speeds.
\end{abstract}

Keywords: Lower bound on achievable data rates, channel state estimation, receiver and transmitter beamforming, multiuser detection, MMSE criterion.

*This work is supported in part by the NSF under Grant No. 0429724. 


\section{Introduction}

In multiuser wireless systems application of multiple antennas appears to be one of the most promising solutions leading to even higher data rates and/or the ability to support greater number of users. Multiuser detection schemes that exploit multiple antennas provide significant increase of the uplink data rates [1]. Likewise, multiple antenna transmitter optimization schemes provide significant increase of the downlink data rates [2-7]. Spatial filtering at the uplink receiver and spatial pre-filtering at the downlink transmitter are examples of linear multiuser detection and transmitter optimization, respectively. In the literature, these specific solutions are also known as receiver and transmitter beamforming.

Both the multiuser detection and transmitter optimization rely heavily on the availability of the channel state information (CSI) at the receiver and transmitter, respectively. Impact of delayed CSI on the downlink data rates is reported in [8]. In systems where the uplink and downlink channel states are mutually independent a CSI feedback is needed to support downlink transmitter optimization. Wireless systems that apply frequency division duplexing (FDD) typically have mutually independent downlink and uplink channel states. Different CSI feedback schemes are proposed and analyzed in [9-11]. In addition, solutions that rely on imperfect or limited CSI feedback are proposed in [12-14].

Unlike the FDD systems, systems with the time division duplexing (TDD) have very high correlation between successive uplink and downlink channel states. Due to the reciprocity of the uplink and downlink channels, in the ideal case of a static environment, the channel states are identical. The above property supports application of the downlink transmitter optimization without an explicit CSI feedback. Specifically, during the uplink transmission interval, the uplink receiver estimates the uplink channel state. Then, during the following downlink transmission interval, the transmitter can apply the channel state estimate to optimize the downlink transmission.

In this study we consider a multiple antenna multiuser TDD system that uses pilot-assisted channel state estimation. Specifically, we consider a wireless system with multiple antennas at the base station and a number of mobile terminals each with a single antenna. We study the per- 
formance of the uplink multiuser detection and downlink transmitter optimization that are based on linear spatial filtering. We analyze how a lower bound on the achievable data rates depends on (1) the arrangement of pilot symbols (preamble or postamble); (2) the duration of the uplink and downlink transmissions; and (3) the percentage power allocated to the pilot. In addition, effects of the terminal speeds are also considered. Our results point to an uplink postamble with flexible percentage (about 20 - $25 \%$ ) pilot power allocation, and transmission durations tailored for specific ranges of terminal speeds.

Most of the published work on the topic of MIMO channel state estimation assumes the blockfading channel model [15-18]. To the best of our knowledge the first results on the achievable data rates for a more realistic correlated fading channel model (Jakes model) were presented in [19]. Similarly, in this work we use a discrete version of a continuously time-varying wireless channel that evolves from symbol to symbol. We believe that this model provides a more accurate description of the temporal evolution of the wireless channel than the block-fading channel model. For example, in TDD systems, a block-fading channel model is not appropriate because it fails to capture the impact of uplink and downlink transmission interval durations on the system performance. More discussions on block-fading versus continuously time-varying wireless channel models can be found in $[19,20]$.

The paper is organized as follows. In Section 2 we present an overview of the system. In Section 3 the uplink model is introduced and the lower bound on the achievable data rates is presented. Equivalent results for the downlink are given in Section 4. The MMSE linear spatial filter is introduced in Section 5 with the corresponding numerical results in Section 6. We conclude in Section 7.

\section{Overview of TDD System and Pilot Arrangement}

We consider a wireless communication system that consists of a base station with $M$ antennas and $N$ mobile terminals (each with a single antenna) as depicted in Figure 1. Furthermore, we assume a TDD system where the uplink and downlink transmissions are assigned to non-overlapping and 
alternating time intervals. Specifically, the uplink and downlink transmissions last $K^{u l}$ and $K^{d l}$ symbols, respectively. For the system with the signal bandwidth $W$, according to the Nyquist rate, the symbol interval is $T_{s}=1 / W$.

During the uplink transmission, $N$ mobile terminals simultaneously transmit signals $x_{k, n}^{u l}$ (corresponding to time instant $k$ and mobile terminal $n$ ). The base station receives the uplink transmissions and performs multiple antenna multiuser detection. Specifically, we focus on linear spatial filtering, i.e., receiver beamforming. The multiuser detection results in $N$ outputs each corresponding to a decision statistics used to decode data transmitted from a particular mobile terminal.

In order to perform the multiuser detection, the base station estimates the uplink channel states. In this particular system we propose a pilot-assisted estimation that relies on the pilot (i.e., reference) symbols that are transmitted by the mobile terminals. To simplify the estimation, the pilots are orthogonal to the data-carrying portion of the signal, and to each other. In Figure 2 we present an example of such an arrangement of the pilot and data symbols where the symbols are time-multiplexed.

Furthermore, the orthogonal pilot arrangement greatly simplifies the channel state estimation procedure. Namely, the estimation between each mobile terminal and the base station can be performed independently from other mobile terminals. In other words, the estimation is identical as in a single user case [21]. If the pilots were not orthogonal either to the data-carrying portion of the transmitted signal or to each other, a more complex joint estimation and/or detection procedure would be required [22].

During the downlink transmission, $N$ independent data streams $x_{k, n}^{d l}$ (corresponding to time instant $k$ and mobile terminal $n$ ) are transmitted on $M$ antennas. Each stream is dedicated to a particular mobile terminal. Before the transmission, the streams are subject to transmitter spatial pre-filtering. Specifically, in this study we focus on linear spatial pre-filtering, i.e., transmitter beamforming.

In order to perform the transmitter optimization, the channel state estimates that are obtained during the preceding uplink transmission interval are applied. Furthermore, to enable the mobile 
terminals to perform decoding of the corresponding data, pilot symbols are embedded within each downlink data stream. The downlink pilot arrangement is identical to the uplink arrangement as is depicted in Figure 2 (where the superscripts ' $u l$ ' should be changed to ' $d l$ ' for the downlink). Note that both the pilot and data-carrying portions of the transmitted signal are filtered by the spatial pre-filter.

While the pilot and data symbol arrangement described above specifically considers time division multiplexing as a means of orthogonalization, the model itself is generic enough in that it captures several transmission scenarios. For example, it is equivalent to either code division or frequency division multiplexing between pilot and data symbols. Moreover, this particular arrangement is directly applicable in single carrier wireless systems with frequency flat fading. In wideband wireless systems, with frequency selective fading, typically OFDM is applied. In that situation, the symbols in Figure 2 can be considered to be transmitted on each OFDM tone (with different data streams transmitted on each tone). If the channel states are correlated between different tones, and there is a priori knowledge about it, a better pilot arrangement may be devised. That case is beyond the scope of this study, and we assume that such a priori knowledge does not exist. A related study on optimal pilot arrangement in single antenna OFDM systems with block Rayleigh fading can be found in [23]. In addition, a detailed treatment of optimal pilot symbol-aided modulation (PSAM) in MIMO frequency-selective channels is given in [24].

\section{Achievable Uplink Data Rates with Pilot-Assisted Chan- nel State Estimation}

The uplink signal received at the base station is presented in a vector form as

$$
\mathbf{y}_{k}^{u l}=\mathbf{H}_{k}^{u l} \mathbf{x}_{k}^{u l}+\mathbf{n}_{k}^{u l}, \quad \mathbf{y}_{k}^{u l} \in \mathcal{C}^{M}, \mathbf{x}_{k}^{u l} \in \mathcal{C}^{N}, \mathbf{n}_{k}^{u l} \in \mathcal{C}^{M}, \mathbf{H}_{k}^{u l} \in \mathcal{C}^{M \times N}
$$

where $\mathbf{x}_{k}^{u l}=\left[x_{k, 1}^{u l}, \cdots, x_{k, N}^{u l}\right]^{\mathrm{T}}$ with $x_{k, n}^{u l}$ being the signal transmitted from mobile terminal $n$. $\mathbf{n}_{k}^{u l}$ is AWGN with $\mathrm{E}\left[\mathbf{n}_{k}^{u l}\left(\mathbf{n}_{k}^{u l}\right)^{\mathrm{H}}\right]=N_{0} \mathbf{I}_{M \times M}$ and $\mathbf{H}_{k}^{u l}$ is the uplink channel matrix, all corresponding to time instant $k$. Further, $h_{k, m, n}^{u l}$ is the $m$ th row and $n$th column element of the matrix $\mathbf{H}_{k}^{u l}$ 
corresponding to the uplink channel state between mobile terminal $n$ and base station antenna $m^{1} . \mathbf{H}_{k}^{u l}$ is a discrete version of the continuously time-varying wireless channel $\mathbf{H}^{u l}(t)$.

The signal transmitted from mobile terminal $n$ (as shown in Figure 2) is

$$
x_{k, n}^{u l}=\underbrace{\sqrt{P_{p}^{u l}} \delta(k-n)}_{\text {Pilot }}+\underbrace{\sum_{i=1}^{K^{u l}-N} \sqrt{P_{d}^{u l}} d_{i, n}^{u l} \delta(k-i-N)}_{\text {Data }}, \text { for } k=1, \cdots, K^{u l}
$$

where $\delta(k)$ is the Kronecker delta function. $d_{i, n}^{u l}$ is a data symbol transmitted from mobile terminal $n$ at time instant $N+i . d_{i, n}^{u l}$ is assumed to be a circularly symmetric complex random variable with Gaussian distribution $\mathcal{N}_{\mathcal{C}}(0,1)$. This assumption on the transmitted signal not only allows us to make certain simplifications in the ensuing analysis, but it has also been shown to be optimal in a number of communication systems and scenarios. For example, its optimality was proven in multiuser MIMO systems in [25].

In the above, the pilot and data symbols are time-multiplexed. Specifically, for mobile terminal $n$, the pilot symbol is sent at the $n$th instant, while the data symbols are sent starting from instant $(N+1)$ and ending with instant $K^{u l}$. Note that during instant $n$, only mobile terminal $n$ transmits the pilot symbol, while the other terminals cease any transmission during that particular instant ${ }^{2}$. The data-carrying interval consists of $K^{u l}-N$ symbols. During that interval the terminals concurrently transmit independent data symbols. In other words, every data-carrying signal dimension is reused $N$ times. Each data symbol is transmitted with the power $P_{d}^{u l}$, while each pilot symbol with the power $P_{p}^{u l}$. The duration of the transmission is limited to $K^{u l}$ symbols, corresponding to the uplink transmission interval.

As shown in Figure 2 (and in the expression (2)), the pilot symbols are transmitted at the beginning of the uplink transmission interval, i.e., forming a preamble. There are also some

\footnotetext{
${ }^{1}$ In this paper, the indices $k$ and $i$ are used to denote different time instant, while the indices $n$ and $l$ are used to denote different mobile terminals. Furthermore, the index $m$ is used to denote the base station antenna.

${ }^{2}$ We assume that there is a synchronization mechanism that enables ideal synchronization between all mobile terminals. Furthermore, we assume that the different propagation delays between each mobile terminal and base station are negligible with respect to the symbol interval or they are compensated via a synchronization scheme that takes the delays into account.
} 
alternative arrangements one can consider. For example, the pilot symbols could be placed in the middle or at the end of the transmission interval corresponding to a midamble or postamble, respectively. The relationship between the transmitted signals with the postamble and preamble is

$$
x_{k, n}^{p o s t-u l}=x_{\left(K^{u l}-k+1\right), n}^{p r e-u l}=x_{\left(K^{u l}-k+1\right), n}^{u l} .
$$

The superscripts post-ul and pre-ul denote the postamble and preamble, respectively, while $x_{k, n}^{u l}$ is given in (2). In the numerical results in Section 6 we will compare the performance for both pilot arrangements.

An estimate of the uplink channel matrix $\mathbf{H}_{k}^{u l}$ is

$$
\widehat{\mathbf{H}}_{k}^{u l}=\mathbf{H}_{k}^{u l}+\mathbf{H}_{e k}^{u l}, \text { for } k=1, \cdots, K^{u l},
$$

where $\mathbf{H}_{e k}^{u l}$ is the estimation error matrix. Its properties will depend on a specific estimation procedure that is described later in the text.

The decision statistics used to detect the data transmitted on the uplink is

$$
\hat{\mathbf{y}}_{k}^{u l}=\mathbf{W}_{k}^{u l} \mathbf{y}_{k}^{u l}, \quad \hat{\mathbf{y}}_{k}^{u l} \in \mathcal{C}^{N}, \mathbf{W}_{k}^{u l} \in \mathcal{C}^{N \times M}, \text { for } k=(N+1), \cdots, K^{u l}
$$

where $\mathbf{W}_{k}^{u l}$ is a spatial filter used to suppress the uplink multiuser interference. The spatial filter is a function of the channel state estimate, i.e., $\mathbf{W}_{k}^{u l}=f\left(\widehat{\mathbf{H}}_{k}^{u l}\right)$. Let us rewrite the above expression as

$$
\hat{\mathbf{y}}_{k}^{u l}=\mathbf{W}_{k}^{u l}\left(\mathbf{H}_{k}^{u l} \mathbf{x}_{k}^{u l}+\mathbf{n}_{k}^{u l}\right)=\mathbf{W}_{k}^{u l} \widehat{\mathbf{H}}_{k}^{u l} \mathbf{x}_{k}^{u l}+\underbrace{\mathbf{W}_{k}^{u l}\left(\mathbf{n}_{k}^{u l}-\mathbf{H}_{e k}^{u l} \mathbf{x}_{k}^{u l}\right)}_{\text {Effective noise vector }}
$$

where the second term is the effective noise vector capturing both the AWGN and noise due to the imperfect knowledge of the true uplink channel state. The decision statistics corresponding to the uplink of mobile terminal $n$ is

$$
\begin{aligned}
\hat{y}_{i, n}^{u l} & =\sqrt{P_{d}^{u l}} d_{i, n}^{u l} \overline{\mathbf{w}}_{k, n}^{u l} \hat{\mathbf{h}}_{k, n}^{u l}+ \\
& +\underbrace{\sum_{l=1, l \neq n}^{N} \sqrt{P_{d}^{u l}} d_{i, l}^{u l} \overline{\mathbf{w}}_{k, n}^{u l} \mathbf{h}_{k, l}^{u l}}_{\text {Multiple access interference }}+
\end{aligned}
$$




$$
\begin{aligned}
& +\underbrace{\overline{\mathbf{w}}_{k, n}^{u l} \mathbf{n}_{k}^{u l}-\sqrt{P_{d}^{u l}} d_{i, n}^{u l} \overline{\mathbf{w}}_{k, n}^{u l} \mathbf{h}_{e k, n}^{u l}}_{\text {Effective noise }}, \\
& \text { for } \quad k=(N+1), \cdots, K^{u l} \text {, and } i=k-N .
\end{aligned}
$$

In the above, $\overline{\mathbf{w}}_{k, n}^{u l}$ is the $n$th row vector of the matrix $\mathbf{W}_{k}^{u l}, \hat{\mathbf{h}}_{k, n}^{u l}$ is the $n$th column vector of the matrix $\hat{\mathbf{H}}_{k}^{u l}, \mathbf{h}_{k, l}^{u l}$ is the $l$ th column vector of the matrix $\mathbf{H}_{k}^{u l}$ and $\mathbf{h}_{e k, n}^{u l}$ is the $n$th column vector of the matrix $\mathbf{H}_{e k}^{u l}$.

As introduced earlier, $d_{i, n}^{u l}$ and $d_{l, n}^{u l}(l=1, \cdots, N$ for $l \neq i)$ are independent circularly symmetric complex random variables with Gaussian distribution $\mathcal{N}_{\mathcal{C}}(0,1)$. Consequently, the multiple access interference in the above equation is equivalent to AWGN. Furthermore, the effective noise has Gaussian distribution but it is not independent of the data $d_{i, n}^{u l}$.

Using the above characteristics we will consider the lower bound on the mutual information between the decision statistics $\hat{y}_{i, n}^{u l}$ and transmitted data $d_{i, n}^{u l}$. It is well known that AWGN is the worst case of noise for mutual information (see $[26,27]$ and references therein). Therefore, the lower bound corresponds to a case when the effective noise is independent of the transmitted data $d_{i, n}^{u l}$, i.e., it is AWGN. Thus,

$$
I\left(D_{i, n}^{u l} ; \hat{Y}_{i, n}^{u l}\right) \geq \log _{2}\left(1+\frac{P_{d}^{u l}\left|\overline{\mathbf{w}}_{k, n}^{u l} \hat{\mathbf{h}}_{k, n}^{u l}\right|^{2}}{\sum_{l=1, l \neq n}^{N} P_{d}^{u l}\left|\overline{\mathbf{w}}_{k, n}^{u l} h_{k, l}^{u l}\right|^{2}+N_{0}\left|\overline{\mathbf{w}}_{k, n}^{u l}\right|^{2}+P_{d}^{u l}\left|\overline{\mathbf{w}}_{k, n}^{u l} \mathbf{h}_{e k, n}^{u l}\right|^{2}}\right) .
$$

Similar arguments for the mutual information lower bound in a scalar multiple access wireless channel and a single user MIMO channel are given in [28] and [16], respectively. The corresponding lower bound on the achievable data rates for mobile terminal $n$ at time instant $k$ is

$$
R_{k, n}^{u l}=\mathrm{E}_{\mathbf{H}_{k}^{u l}, \hat{\mathbf{H}}_{k}^{u l}, \mathbf{H}_{e k}^{u l}}\left[\log _{2}\left(1+\frac{P_{d}^{u l}\left|\overline{\mathbf{w}}_{k, n}^{u l} \hat{\mathbf{h}}_{k, n}^{u l}\right|^{2}}{\sum_{l=1, l \neq n}^{N} P_{d}^{u l}\left|\overline{\mathbf{w}}_{k, n}^{u l} \mathbf{h}_{k, l}^{u l}\right|^{2}+N_{0}\left|\overline{\mathbf{w}}_{k, n}^{u l}\right|^{2}+P_{d}^{u l}\left|\overline{\mathbf{w}}_{k, n}^{u l} h_{e k, n}^{u l}\right|^{2}}\right)\right] .
$$

Considering the whole uplink transmission interval, the lower bound on the achievable uplink data 
rates for mobile terminal $n$ can be given as

$$
C_{n}^{u l} \geq R_{n}^{u l}=\frac{1}{K^{u l}} \sum_{k=N+1}^{K^{u l}} R_{k, n}^{u l} .
$$

The above expression explicitly accounts for the fact that out of the $K^{u l}$ available signal dimensions, $N$ signal dimensions are used for the pilots. Note that in the above expression, equality holds if the effective noise is AWGN. If the effective noise is not AWGN, then the above rate represents the worst case scenario, i.e., the lower bound. In achieving the above rate, the receiver assumes that the effective noise is independent of the transmitted data with Gaussian spatially white distribution.

Note that the expressions in (8) and (10) are valid for any linear multiuser detection scheme and any pilot-assisted channel state estimation scheme. Selection of a specific linear multiuser detector and channel state estimation scheme will only affect statistical properties of the quantities in (9). In fact knowing these properties allows us to numerically obtain the rates given in (9) and (10).

\section{Uplink Channel State Estimation}

We now describe the channel state estimation procedure and the corresponding properties of the estimation error matrix $\mathbf{H}_{e k}^{u l}$ in (4).

Using the pilot-assisted estimation, at time instant $k=n$ the estimate of the uplink channel state between mobile terminal $n$ and base station antenna $m$ is

$$
\hat{h}_{n, m, n}^{u l}=h_{n, m, n}^{u l}+e_{n, m, n}^{u l}
$$

where the first index in the subscript denotes the time instant, the second index denotes the base station antenna while the third index denotes the mobile terminal. $e_{n, m, n}^{u l}$ is the estimation noise at time instant $n$. It is modeled as a random variable with complex Gaussian distribution $\mathcal{N}_{\mathcal{C}}\left(0, N_{0} / P_{p}^{u l}\right)$. We assume that the uplink receiver will use the above estimate for the duration of the uplink transmission. In other words,

$$
\hat{h}_{k, m, n}^{u l}=\hat{h}_{n, m, n}^{u l}=h_{k, m, n}^{u l}+h_{e k, m, n}^{u l}, \text { for } k=1, \cdots, K^{u l}
$$


where $h_{e k, m, n}^{u l}$ is a difference, i.e., estimation error between the true channel state $h_{k, m, n}^{u l}$ and $\hat{h}_{n, m, n}^{u l}$, which is the channel state assumed by the uplink receiver. Using the estimate in (11), the above estimation error becomes

$$
h_{e k, m, n}^{u l}=h_{n, m, n}^{u l}-h_{k, m, n}^{u l}+e_{n, m, n}^{u l} .
$$

Note that $h_{e k, m, n}^{u l}$ is actually $m$ th row and $n$th column entry of the matrix $\mathbf{H}_{e k}^{u l}$ in (4) for $k=$ $1, \cdots, K^{u l}, m=1, \cdots, M$, and $n=1, \cdots, N$.

Using the statistical properties of the estimation error outlined above, we can numerically obtain the rate $R_{n}^{u l}$ in (10). It will represent the achievable data rate for reliable transmission (error free) for this specific estimation procedure. Using a better channel state estimation scheme (e.g., a decision-driven scheme) may result in higher achievable data rates. Further, channel state prediction may also provide further improvements (see for example [29] and references therein).

\section{Achievable Downlink Data Rates with Pilot-Assisted Channel State Estimation}

The downlink signal received at the mobile terminals is presented in a vector form as

$$
\mathbf{y}_{k}^{d l}=\mathbf{H}_{k}^{d l} \mathbf{W}_{k}^{d l} \mathbf{x}_{k}^{d l}+\mathbf{n}_{k}^{d l}, \quad \mathbf{y}_{k}^{d l} \in \mathcal{C}^{N}, \mathbf{x}_{k}^{d l} \in \mathcal{C}^{N}, \mathbf{n}_{k}^{d l} \in \mathcal{C}^{N}, \mathbf{H}_{k}^{d l} \in \mathcal{C}^{N \times M}, \mathbf{W}_{k}^{d l} \in \mathcal{C}^{M \times N},
$$

where the $n$th component of the vector $\mathbf{y}_{k}^{d l}$ is the signal received at mobile terminal $n$. Furthermore, $\mathbf{x}_{k}^{d l}=\left[x_{k, 1}^{d l}, \cdots, x_{k, N}^{d l}\right]^{\mathrm{T}}$ with $x_{k, n}^{d l}$ being a signal transmitted to mobile terminal $n$, all corresponding to time instant $k$. Furthermore, $\mathbf{W}_{k}^{d l}$ is a spatial pre-filter applied at the base station with the constraint

$$
\left\|\mathbf{W}_{k}^{d l}\right\|^{2}=N
$$

where $\|$.$\| is the Frobenius norm.$

After changing the superscripts ' $u l$ ' to ' $d l$ ', the definition given in the equation (2) is directly applicable to the downlink. In addition, the downlink transmission starts immediately upon completion of the uplink transmission, thus, $k=\left(K^{u l}+1\right), \cdots,\left(K^{u l}+K^{d l}\right)$. As said earlier, both 
the pilot and data-carrying portions of the transmitted signal are filtered by the spatial pre-filter $\mathbf{W}_{k}^{d l}$.

The application of the spatial pre-filter results in a composite downlink channel matrix $\mathbf{G}_{k}^{d l}$ given as

$$
\mathbf{G}_{k}^{d l}=\mathbf{H}_{k}^{d l} \mathbf{W}_{k}^{d l}, \quad \mathbf{G}_{k}^{d l} \in \mathcal{C}^{N \times N}
$$

An estimate of the composite downlink channel matrix $\mathbf{G}_{k}^{d l}$ is

$$
\widehat{\mathbf{G}}_{k}^{d l}=\mathbf{G}_{k}^{d l}+\mathbf{G}_{e k}^{d l}
$$

where $\mathbf{G}_{e k}^{d l}$ is the estimation error matrix. Only the diagonal entries of this estimation error matrix are of interest in this study. Their properties will be specified later in the text.

The decision statistics corresponding to the downlink of mobile terminal $n$ is

$$
\begin{gathered}
\hat{y}_{i, n}^{d l}=\sqrt{P_{d}^{d l}} d_{i, n}^{d l} \hat{g}_{k, n, n}^{d l}+\underbrace{\sum_{l=1, l \neq n}^{N} \sqrt{P_{d}^{d l}} d_{i, l}^{d l} g_{k, n, l}^{d l}}_{\text {Interference }}+\underbrace{n_{k, n}^{d l}-\sqrt{P_{d}^{d l}} d_{i, n}^{d l} g_{e k, n, n}^{d l}}_{\text {Effective noise }}, \\
\text { for } \quad k=\left(K^{u l}+N+1\right), \cdots,\left(K^{u l}+K^{d l}\right), \text { and } i=k-K^{u l}-N .
\end{gathered}
$$

In the above, $\hat{g}_{k, n, n}^{d l}$ is the $n$th diagonal element of the matrix $\widehat{\mathbf{G}}_{k}^{d l}, g_{k, n, l}^{d l}$ is the $n$th row and $l$ th column element of the matrix $\mathbf{G}_{k}^{d l}$ and $g_{e k, n, n}^{d l}$ is the $n$th diagonal element of the matrix $\mathbf{G}_{e k}^{d l}$.

Similar to the uplink case, a lower bound on the downlink data rates for mobile terminal $n$ is,

$$
C_{n}^{d l} \geq R_{n}^{d l}=\frac{1}{K^{d l}} \sum_{k=K^{u l}+N+1}^{K^{u l}+K^{d l}} R_{k, n}^{d l}
$$

for

$$
R_{k, n}^{d l}=\mathrm{E}_{\mathbf{G}_{k}^{d l}, \widehat{\mathbf{G}}_{k}^{d l}, \mathbf{G}_{e k}^{d l}}\left[\log _{2}\left(1+\frac{P_{d}^{d l}\left|\hat{g}_{k, n, n}^{d l}\right|^{2}}{\sum_{l=1, l \neq n}^{N} P_{d}^{d l}\left|g_{k, n, l}^{d l}\right|^{2}+N_{0}+P_{d}^{d l}\left|g_{e k, n, n}^{d l}\right|^{2}}\right)\right]
$$

Expression (19) explicitly accounts for the fact that out of the $K^{d l}$ available signal dimensions, $N$ signal dimensions are used for the pilots. As in the case of the uplink data rates, if the effective noise is not AWGN, then the above rate represents the worst case scenario, i.e., the lower bound. 
Similar to the results in (10) and (9), the results in (19) and (20) are valid for the case of any linear spatial pre-filtering and pilot-assisted channel state estimation scheme.

\section{Downlink Channel State Estimation}

We now describe the downlink channel state estimation procedure and the corresponding properties of the diagonal entries $g_{e k, n, n}^{d l}$ of the estimation error matrix $\mathbf{G}_{e k}^{d l}$ in (17), for $n=1, \cdots, N$.

Using the pilot-assisted estimation, at time instant $i=\left(n+K^{u l}\right)$ an estimate of the composite downlink channel state between mobile terminal $n$ and the base station is

$$
\hat{g}_{i, n, n}^{d l}=g_{i, n, n}^{d l}+e_{i, n, n}^{d l}, \text { for } i=\left(n+K^{u l}\right)
$$

where the first index in the subscript denotes the time instant, while the second and third indices stand for coordinates of the diagonal entry of the corresponding matrix. $e_{i, n, n}^{d l}$ is the estimation noise at time instant $i=\left(n+K^{u l}\right)$. It is modeled as a random variable with complex Gaussian distribution $\mathcal{N}_{\mathcal{C}}\left(0, N_{0} / P_{p}^{d l}\right)$. We assume that mobile terminal $n$ will use the above estimate for the duration of the downlink transmission. In other words,

$$
\hat{g}_{k, n, n}^{d l}=\hat{g}_{i, n, n}^{d l}=g_{k, n, n}^{d l}+g_{e k, n, n}^{d l}, \text { for } k=\left(K^{u l}+1\right), \cdots,\left(K^{u l}+K^{d l}\right), \text { and } i=\left(n+K^{u l}\right),
$$

where $g_{e k, n, n}^{d l}$ is a difference, i.e., estimation error between the true composite channel state $g_{k, n, n}^{d l}$ and $\hat{g}_{i, n, n}^{d l}$, which is the channel state assumed by mobile terminal $n$.

Using the estimate in (21), the estimation error becomes

$$
g_{e k, n, n}^{d l}=g_{i, n, n}^{d l}-g_{k, n, n}^{d l}+e_{i, n, n}^{d l}=\overline{\mathbf{h}}_{i, n}^{d l} \mathbf{w}_{i, n}^{d l}-\overline{\mathbf{h}}_{k, n}^{d l} \mathbf{w}_{k, n}^{d l}+e_{i, n, n}^{d l}
$$

where $\overline{\mathbf{h}}_{i, n}^{d l}$ and $\overline{\mathbf{h}}_{k, n}^{d l}$ are the $n$th row vectors of the downlink channel matrices $\mathbf{H}_{i}^{d l}$ and $\mathbf{H}_{k}^{d l}$, respectively. Further, $\mathbf{w}_{i, n}^{d l}$ and $\mathbf{w}_{k, n}^{d l}$ are the $n$th column vectors of the matrices $\mathbf{W}_{i}^{d l}$ and $\mathbf{W}_{k}^{d l}$, respectively. $g_{e k, n, n}^{d l}$ is the $n$th diagonal entry of the matrix $\mathbf{G}_{e k}^{d l}$ in $(17)$ for $k=\left(K^{u l}+1\right), \cdots,\left(K^{u l}+\right.$ $\left.K^{d l}\right), i=\left(n+K^{u l}\right)$ and $n=1, \cdots, N$. 
Using the above properties of the estimation error we can numerically obtain the rate $R_{n}^{d l}$ in (19). It will represent the achievable data rate for this specific estimation procedure. Using a better channel state estimation or prediction scheme may result in higher achievable data rates.

\section{Illustration of Uplink and Downlink Spatial Filtering}

In general, the above results on the achievable data rates in (10) and (19) are applicable for arbitrary uplink and downlink linear spatial filter $\mathbf{W}_{k}^{u l}$ and $\mathbf{W}_{k}^{d l}$, respectively. In this section we present one example of each of these.

For the uplink, the base station assumes that $\mathbf{H}_{k}^{u l}=\widehat{\mathbf{H}}_{k}^{u l}$, i.e., it ignores the fact that $\mathbf{H}_{k}^{u l} \neq \widehat{\mathbf{H}}_{k}^{u l}$, all for time instant $k$. The uplink linear multiuser detector is

$$
\mathbf{W}_{k}^{u l}=\left(\left(\widehat{\mathbf{H}}_{k}^{u l}\right)^{\mathrm{H}} \widehat{\mathbf{H}}_{k}^{u l}+\frac{N_{0}}{P_{d}^{u l}} \mathbf{I}\right)^{-1}\left(\widehat{\mathbf{H}}_{k}^{u l}\right)^{\mathrm{H}} .
$$

Note that in the case of $\mathbf{H}_{k}^{u l}=\widehat{\mathbf{H}}_{k}^{u l}$, the above spatial filter minimizes the mean square error, i.e., it is the linear MMSE multiuser detector. It is well known that the MMSE receiver is the optimal linear receiver that maximizes the received $S I N R$ (and rate) for each user on the uplink [30,31].

Due to the reciprocity between the uplink and downlink channels, the downlink transmitter assumes that $\mathbf{H}_{k}^{d l}=\left(\mathbf{H}_{k}^{u l}\right)^{\mathrm{T}}$ and that their estimates are $\widehat{\mathbf{H}}_{k}^{d l}=\left(\widehat{\mathbf{H}}_{k}^{u l}\right)^{\mathrm{T}}$. In this particular example the mismatch between the true downlink and estimated uplink channel states is ignored. The downlink transmitter optimization results in a linear spatial pre-filter

$$
\mathbf{W}_{k}^{d l}=\mathbf{U}_{k} \mathbf{P}_{k}=\left(\widehat{\mathbf{H}}_{k}^{d l}\right)^{\mathrm{H}}\left(\widehat{\mathbf{H}}_{k}^{d l}\left(\widehat{\mathbf{H}}_{k}^{d l}\right)^{\mathrm{H}}+\frac{N_{0}}{P_{d}^{d l}} \mathbf{I}\right)^{-1} \mathbf{P}_{k}, \text { for } \widehat{\mathbf{H}}_{k}^{d l}=\left(\widehat{\mathbf{H}}_{k}^{u l}\right)^{\mathrm{T}}
$$

In the above, the matrix $\mathbf{P}_{k}$ is a diagonal matrix such that the constraint in (15) is satisfied. Specifically, the $n$th diagonal element of $\mathbf{P}_{k}$ is selected as

$$
p_{k, n, n}=\sqrt{\frac{\gamma_{k, n}}{\mathbf{u}_{k, n}^{\mathrm{H}} \mathbf{u}_{k, n}}}=\frac{\sqrt{\gamma_{k, n}}}{\left|\mathbf{u}_{k, n}\right|}
$$

where $\mathbf{u}_{k, n}$ is the $n$th column vector of the matrix $\mathbf{U}_{k}$ in $(25)$, for $n=1, \cdots, N$.

We now consider an idealized case where (i) the uplink and downlink channels are identical (i.e., $\mathbf{H}_{k}^{d l}=\left(\mathbf{H}_{k}^{u l}\right)^{\mathrm{T}}$ ), (ii) the channel states are perfectly known (i.e., $\mathbf{H}_{k}^{u l}=\widehat{\mathbf{H}}_{k}^{u l}$ and $g_{k, n, n}^{d l}=\hat{g}_{k, n, n}^{d l}$ ), 
(iii) the transmit powers on the uplink and downlink are identical (i.e., $P_{d}^{u l}=P_{d}^{d l}$ ) as well as (iv) the transmission intervals (i.e., $K^{u l}=K^{d l}$ ). For the above idealizations, the quantity $\gamma_{k, n}$ in $(26)$ can be determined such that the multiuser detector in (24) and transmitter spatial pre-filter in (25) achieve identical uplink and downlink data rates, $R_{k, n}^{u l}=R_{k, n}^{d l}$, for $n=1, \cdots, N$. The details are given in Appendix A.

Note that in the above example, the spatial filters are design under the assumption that the channel state estimates are identical to the true channel states. Further improvements can be realized by designing the spatial filters that optimally account for the mismatch between the true and estimated channel states. This may be a subject of our future work.

\section{$6 \quad$ Numerical results}

In the following numerical results the channel between each base station and mobile terminal antenna is modeled as a complex Gaussian unit variance random variable with the zero mean ${ }^{3}$. Furthermore, the number of the base station antennas is chosen to be $M=4$ and the mobile terminals $N=4$. The carrier frequency is $f_{c}=2 \mathrm{GHz}$, and the signal bandwidth $W=15.625$ $\mathrm{KHz}$ (which is a typical bandwidth of an OFDM tone in the WiMAX-2004 wireless standard). The Jakes model is used to model the temporal evolution of the channel state [32]. For example, the uplink channel state between mobile terminal $n$ and base station antenna $m$ evolves as

$$
h_{k, m, n}^{u l}=\frac{1}{\sqrt{L}} \sum_{l=0}^{L-1} e^{j\left(2 \pi f_{n}^{d} \cos (2 \pi l / L) T_{s} k+\phi_{l, m, n}\right)}
$$

where $f_{n}^{d}$ is the Doppler frequency $\left(f_{n}^{d}=f_{c} v_{n} / c\right)$, with $v_{n}$ being the speed of mobile terminal $n$ and $c$ is the speed of light. Further, $T_{s}$ is the symbol interval, and according to the Nyquist rate it is $T_{s}=1 / W . \phi_{l, m, n}$ is a random phase shift (generated as $\mathcal{U}[02 \pi]$ ). We set $L=100$.

We will observe the performance of the system with respect to the amount of transmitted energy that is allocated to the pilot (percentage wise). For example, in the uplink case this

\footnotetext{
${ }^{3}$ Note that this may correspond to a case when a power control mechanism eliminates effects of large scale fading (i.e., path loss and shadowing) allowing statistically identical and independent small scale fading between the base station and mobile terminals (which is captured by the Rayleigh channel model).
} 
percentage is denoted as $\mu^{u l}$ and is given as

$$
\mu^{u l}=\frac{P_{p}^{u l}}{\left(K^{u l}-N\right) P_{d}^{u l}+P_{p}^{u l}} 100[\%],
$$

while the average transmit power is

$$
P^{u l}=\frac{\left(K^{u l}-N\right) P_{d}^{u l}+P_{p}^{u l}}{K^{u l}}
$$

Equivalent expression can be derived for the downlink as well.

In Figure 3 we present the sum (across all mobile terminals) of the uplink and downlink data rates in (9) and (20) (for the percentage pilot power $\mu=\mu^{u l}=\mu^{d l}=20 \%$ and $S N R=$ $\left.10 \log P^{u l} / N_{0}=10 \log P^{d l} / N_{0}=10 \mathrm{~dB}\right)$. The results are presented for different mobile terminal speeds $v_{n}=3,30$ and $90 \mathrm{kmph}$, for $n=1, \cdots, N$. The uplink and downlink transmission intervals span symbols 1 to 50 and 51 to 100, respectively. The uplink and downlink pilot symbols are transmitted as a preamble at the beginning of the corresponding transmission intervals. Therefore, the rates are zero for symbols 1 to 4 and 51 to 54. High rates are experienced within few symbols away from the uplink pilot. As the distance in time increases away from the uplink pilot symbols, the rates get lower. This is because the uplink channel state estimates are getting obsolete, thus diminishing the data rates. Note that the downlink data rates are particularly low.

In Figure 4 we assess performance of the alternative uplink pilot arrangement, where the pilot symbols are sent at the end of the uplink transmission interval forming the uplink postamble. Therefore the uplink rate is zero for symbols 47 to 50. For the uplink, the performance is a mirror image of the case with the preamble. However, a dramatic improvement is noted for the downlink. This is because placing the uplink pilot symbols at the end of the uplink transmission interval allows the downlink transmitter pre-filtering to apply channel state estimates that are recent.

In Figure 5 we present the sum of the uplink and downlink data rates in (10) and (19) as a function of the transmission interval duration $K=K^{u l}=K^{d l}$ (for the percentage pilot power $\mu=20 \%$ and $S N R=10 \mathrm{~dB}$ ). For example, a short transmission interval may provide frequent channel state estimates, but relative pilot overhead will grow. In general, for a given terminal speeds, there is an optimal duration of the interval. Similarly, in Figure 6 we present the sum of 
the data rates as a function of the percentage pilot power $\mu$ (for $K=30$ and $S N R=10 \mathrm{~dB}$ ). From the results, we note that the rates have a broad maximum, and the system is not sensitive to exact selection of the pilot power. In Figure 7 we present the sum of the downlink data rates in (19) as a function of both the transmission interval duration and the percentage pilot power. We note that the function is concave for the given range of the parameters. Based on these results, for example, selecting $K=30$ and $\mu=20 \%$ will accommodate a fairly wide range of terminal speeds.

In Figure 8 we present the sum of the uplink and downlink data rates in (10) and (19) as a function of $S N R$ (for $K=30$ and $\mu=20 \%$ ). The idealized case corresponds the data rates where the uplink receiver and downlink transmitter have ideal knowledge of the channel states (without any pilot overhead) and are in fact equal. In the case of the pilot-assisted channel state estimation, the data rates exhibit a ceiling (interference limited behavior). Thus, contrary to the idealized case, the data rates cease to increase beyond a certain $S N R$. Note that a similar behaviour is predicted by a general information theoretic analysis in [27]. Unlike [27], here we consider a more practical pilot-assisted channel state estimation and linear spatial filtering scheme.

\section{Conclusions}

In this paper we have presented a multiple antenna multiuser TDD system that uses pilot-assisted channel state estimation. We have analyzed the performance of the uplink multiuser detection and downlink transmitter optimization that are based on linear spatial filtering. Using a discrete version of the continuously time-varying wireless channel we have analyzed how a lower bound on the achievable data rates depends on the arrangement of pilot symbols, the duration of the uplink and downlink transmissions and the percentage power allocated to the pilot. We have shown that placing pilot symbols at the end of the uplink transmission interval can improve the downlink data rates. Further, allocating about $20-25 \%$ of the total power budget for the pilot seems like a robust choice over a wide range of channel conditions and terminal speeds. In addition, the analysis presented here can be used to tailor the duration of the uplink and downlink transmissions 
for specific ranges of terminal speeds.

Note that in designing the spatial filters we have assumed that the channel state estimates are identical to the true channel states. Design of the spatial filters that optimally account for the mismatch between the true and estimated channel states may be a subject of future work. In addition, we have considered the effects of a simple channel state estimation procedure. Consequently, the design and performance analysis of more advanced channel state estimation and prediction schemes are also of future interest. Furthermore, a number of results in this paper are based on simulations and future work could augment this with additional analysis.

\section{A Appendix}

Consider an idealized case where (i) the uplink and downlink channels are identical (i.e., $\mathbf{H}_{k}^{d l}=$ $\left(\mathbf{H}_{k}^{u l}\right)^{\mathrm{T}}$ ), (ii) the channel states are perfectly known (i.e., $\mathbf{H}_{k}^{u l}=\widehat{\mathbf{H}}_{k}^{u l}$ and $g_{k, n, n}^{d l}=\hat{g}_{k, n, n}^{d l}$ ), (iii) the transmit powers on the uplink and downlink are identical (i.e., $P_{d}^{u l}=P_{d}^{d l}$ ) as well as (iv) the transmission intervals (i.e., $K^{u l}=K^{d l}$ ). We now present details on how to determine the quantities $\gamma_{k n}$ in (26) such that the multiuser detector in (24) and transmitter spatial pre-filter in (25) achieve identical uplink and downlink data rates, $R_{k, n}^{u l}=R_{k, n}^{d l}$, for $n=1, \cdots, N$. The multiuser detector in (24) becomes

$$
\mathbf{W}_{k}^{u l}=\left(\left(\mathbf{H}_{k}^{u l}\right)^{\mathrm{H}} \mathbf{H}_{k}^{u l}+\frac{N_{0}}{P_{d}^{u l}} \mathbf{I}\right)^{-1}\left(\mathbf{H}_{k}^{u l}\right)^{\mathrm{H}} .
$$

Likewise, for $\mathbf{H}_{k}^{d l}=\left(\mathbf{H}_{k}^{u l}\right)^{\mathrm{T}}$ the transmitter spatial pre-filter in (25) becomes

$$
\mathbf{W}_{k}^{d l}=\left(\mathbf{H}_{k}^{d l}\right)^{\mathrm{H}}\left(\mathbf{H}_{k}^{d l}\left(\mathbf{H}_{k}^{d l}\right)^{\mathrm{H}}+\frac{N_{0}}{P_{d}^{d l}} \mathbf{I}\right)^{-1} \mathbf{P}_{k} .
$$

Furthermore, for $P_{d}^{d l}=P_{d}^{u l}$

$$
\mathbf{W}_{k}^{d l}=\left(\mathbf{W}_{k}^{u l}\right)^{\mathrm{T}} \mathbf{P}_{k}
$$

For the $n$th mobile terminal, the uplink signal to interference and noise power ratio $(S I N R)$ is

$$
S I N R_{k, n}^{u l}=\frac{P_{d}^{u l}\left|\overline{\mathbf{w}}_{k, n}^{u l} \mathbf{h}_{k, n}^{u l}\right|^{2}}{\sum_{l=1, l \neq n}^{N} P_{d}^{u l}\left|\overline{\mathbf{w}}_{k, n}^{u l} \mathbf{h}_{k, l}^{u l}\right|^{2}+N_{0}\left|\overline{\mathbf{w}}_{k, n}^{u l}\right|^{2}}
$$


where $\overline{\mathbf{w}}_{k, n}^{u l}$ is the $n$th row vector of the matrix $\mathbf{W}_{k}^{u l}$ while $\mathbf{h}_{k, n}^{u l}$ is the $n$th column and $\mathbf{h}_{k, l}^{u l}$ is the lth column vector of the matrix $\mathbf{H}_{k}^{u l}$.

For the spatial pre-filtering in (31), the corresponding downlink $S I N R$ is

$$
S I N R_{k, n}^{d l}=\frac{P_{d}^{d l}\left|\overline{\mathbf{h}}_{k, n}^{d l} \mathbf{w}_{k, n}^{d l}\right|^{2}}{\sum_{l=1, l \neq n}^{N} P_{d}^{d l}\left|\overline{\mathbf{h}}_{k, n}^{d l} \mathbf{w}_{k, l}^{d l}\right|^{2}+N_{0}}
$$

where $\overline{\mathbf{h}}_{k, n}^{d l}$ is the $n$th row vector the matrix $\mathbf{H}_{k}^{d l} \cdot \mathbf{w}_{k, n}^{d l}$ is the $n$th column and $\mathbf{w}_{k, l}^{d l}$ is the $l$ th column vector of the matrix $\mathbf{W}_{k}^{d l}$. Based on the assumptions that led to the result in (32), we can rewrite the above $S I N R_{k, n}^{d l}$ as

$$
S I N R_{k, n}^{d l}=\frac{\gamma_{k, n} P_{d}^{u l}\left|\overline{\mathbf{w}}_{k, n}^{u l} \mathbf{h}_{k, n}^{u l}\right|^{2} /\left|\overline{\mathbf{w}}_{k, n}^{u l}\right|^{2}}{\sum_{l=1, l \neq n}^{N} \gamma_{k, l} P_{d}^{u l}\left|\overline{\mathbf{w}}_{k, l}^{u l} \mathbf{h}_{k, n}^{u l}\right|^{2} /\left|\overline{\mathbf{w}}_{k, l}^{u l}\right|^{2}+N_{0}} .
$$

As a design criterion, our goal is to determine the quantities $\gamma_{k, n}$ that will result in the identical uplink and downlink rates, $R_{k, n}^{u l}=R_{k, n}^{d l}$, for $n=1, \cdots, N$. We define the following set of equations,

$$
S I N R_{k, n}^{d l}=S I N R_{k, n}^{u l}, \text { for } n=1, \cdots, N
$$

They lead to the identical uplink and downlink rates. In addition, the equations are linear.

Let us now define a matrix $\mathbf{T}_{k}$ with the following entries

$$
t_{k, n, n}=\sum_{l=1, l \neq n}^{N} \frac{\left|\overline{\mathbf{w}}_{k n}^{u l} \mathbf{h}_{k l}^{u l}\right|^{2}}{\left|\overline{\mathbf{w}}_{k, n}^{u l}\right|^{2}}+\frac{N_{0}}{P_{d}^{u l}}
$$

and

$$
t_{k, n, l}=-\frac{\left|\overline{\mathbf{w}}_{k, l}^{u l} \mathbf{h}_{k, n}^{u l}\right|^{2}}{\left|\overline{\mathbf{w}}_{k, l}^{u l}\right|^{2}}, \quad l \neq n,
$$

for $n=1, \cdots, N$ and $l=1, \cdots, N$. Furthermore we define a vector $\mathbf{v}_{k}$ with the following entries

$$
v_{k, n}=\frac{N_{0}}{P_{d}^{u l}}, \text { for } n=1, \cdots, N
$$

Using the above definitions, the system of linear equations in (36) can be expressed as

$$
\mathbf{T}_{k} \gamma_{k}=\mathbf{v}_{k}
$$

where $\gamma_{k}=\left[\gamma_{k, 1} \cdots \gamma_{k, N}\right]^{\mathrm{T}}$. Thus, the solution is

$$
\gamma_{k}=\left[\gamma_{k, 1} \cdots \gamma_{k, N}\right]^{\mathrm{T}}=\mathbf{T}_{k}^{-1} \mathbf{v}_{k}
$$


Furthermore, based on the equations in (36) it can be shown that

$$
\gamma_{k, 1}+\cdots+\gamma_{k, N}=N
$$

which satisfies the constraint in (15) with equality. The above result can be obtained by summing column elements of the vectors on both sides of the equation (40). 


\section{References}

[1] J. H. Winters, J. Salz, and R. D. Gitlin, "The impact of antenna diversity on the capacity of wireless communication systems," IEEE Transactions on Communications, vol. 42, pp. 1740-1751, February-April 1994.

[2] G. Caire and S. Shamai, "On the achievable throughput of a multiantenna Gaussian broadcast channel," IEEE Transactions on Information Theory, vol. 49, pp. 1691-1706, July 2003.

[3] D. Tse and P. Viswanath, "On the capacity region of the vector Gaussian broadcast channel," IEEE International Symposium on Information Theory, pp. 342-342, July 2003.

[4] E. Rashid-Farrohi, L. Tassiulas, and K. Liu, "Joint optimal power control and beamforming in wireless networks using antenna arrays," IEEE Transactions on Communications, vol. 46, pp. 1313-1323, October 1998.

[5] E. Visotsky and U. Madhow, "Optimum beamforming using transmit antenna arrays," IEEE Vehicular Technology Conference, vol. 1, pp. 851-856, May 1999.

[6] K. Pedersen, P. Mogensen, and J. Ramiro-Moreno, "Application and performance of downlink beamforming techniques in UMTS," IEEE Communications Magazine, pp. 134-143, October 2003.

[7] D. Samardzija and N. Mandayam, "Multiple antenna transmitter optimization schemes for multiuser systems," IEEE Vehicular Technology Conference, pp. 399-403, October 2003.

[8] D. Samardzija, N. Mandayam, and D. Chizhik, "Adaptive transmitter optimization in multiuser multiantenna systems: theoretical limits, effect of delays and performance enhancements," EURASIP Journal on Wireless Communications and Networking, pp. 298-307, August 2005.

[9] D. J. Love and R. W. Heath, "Grassmannian beamforming for multiple-input multiple-output wireless systems," IEEE Transaction on Information Theory, vol. 49, pp. 2735-2747, October 2003.

[10] D. Samardzija and N. Mandayam, "Unquantized and uncoded channel state information feedback in multiple antenna multiuser systems," IEEE Transactions on Communication, vol. 54, pp. 1335-1345, July 2006.

[11] T. Yoo, N. Jindal, and A. Goldsmith, "Finite-rate feedback MIMO broadcast channels with a large number of users," IEEE International Symposium on Information Theory, 2006.

[12] E. Visotsky and U. Madhow, "Space-time transmit precoding with imperfect feedback," IEEE Transactions on Information Theory, vol. 47, pp. 2632-2639, September 2001.

[13] S. Zhou and G. B. Giannakis, "Adaptive modulation for multi-antenna transmissions with channel mean feedback," IEEE Transactions on Wireless Communications, vol. 3, pp. 1626-1636, September 2004. 
[14] P. Xia, S. Zhou, and G. B. Giannakis, "Multi-antenna adaptive modulation with beamforming based on bandwidth-constrained feedback," IEEE Transactions on Communications, vol. 53, pp. 526-536, March 2005.

[15] T. L. Marzetta, "Blast training: estimating channel characteristics for high-capacity space-time wireless," 37th Annual Allerton Conference on Communications, Control and Computing, September 1999.

[16] B. Hassibi and B. M. Hochwald, "How much training is needed in multiple-antenna wireless links," IEEE Transactions on Information Theory, vol. 49, pp. 951-963, April 2003.

[17] D. Samardzija and N. Mandayam, "Pilot assisted estimation of MIMO fading channel response and achievable data rates," IEEE Transactions on Signal Processing, vol. 51, pp. 2882-2890, November 2003.

[18] D. Samardzija, L. Xiao, and N. Mandayam, "Impact of pilot assisted channel state estimation on multiple antenna multiuser TDD systems with spatial filtering," Conference on Information Sciences and Systems, March 2006.

[19] J. Baltersee, G. Fock, and H. Meyr, "Achievable rate of MIMO channels with data-aided channel estimation and perfect interleaving," IEEE JSAC, vol. 19, pp. 2358-2368, December 2001.

[20] J. Baltersee, G. Fock, and H. Meyr, "An information theoretic foundation of synchronized detection," IEEE Transactions on Communication, vol. 49, pp. 2115-2123, December 2001.

[21] H. V. Poor, An Introduction to Signal Detection and Estimation. Springer-Verlag, second ed., 1994.

[22] M. Loncar, R. Muller, T. Abe, and J. Wehinger, "Iterative joint detection, decoding, and channel estimation for dual antenna arrays in frequency selective fading," International Symposium on Wireless Personal Multimedia Communications, October 2002.

[23] S. Ohno and G. B. Giannakis, "Capacity maximizing MMSE-optimal pilots for wireless OFDM over frequencyselective block Rayleigh-fading channels," IEEE Transactions on Information Theory, vol. 50, pp. 2138-2145, September 2005.

[24] M. Xiaoli, Y. Liuqing, and G. B. Giannakis, "Optimal training for MIMO frequency-selective fading channels," IEEE Transactions on Wireless Communications, vol. 4, pp. 453-466, March 2005.

[25] H. Weingarten, Y. Steinberg, and S. Shamai, "The capacity region of the Gaussian multiple-input multipleoutput broadcast channel," IEEE Transactions on Information Theory, vol. 52, pp. 3936-3964, September 2006.

[26] R. G. Gallager, Information Theory and Reliable Communications. New York: John Wiley and Sons, 1968.

[27] A. Lapidoth, S. Shamai, and M. A. Wigger, "On the capacity of fading MIMO broadcast channels with imperfect transmitter side-information," http://www.arxiv.org/pdf/cs.IT/0605079, May 2006. 
[28] M. Medard, "The effect upon channel capacity in wireless communication of perfect and imperfect knowledge of the channel," IEEE Transactions on Information Theory, vol. 46, pp. 933-946, May 2000.

[29] T. Svantesson and A. L. Swindlehurst, "A performance bound for prediction of MIMO channels," IEEE Transactions on Signal Processing, vol. 54, pp. 520-529, February 2006.

[30] U. Madhow and M. Honig, "MMSE interference suppression for direct-sequence spread-spectrum CDMA," IEEE Transactions on Communications, vol. 42, pp. 3178-3188, December 1994.

[31] S. Verdú, Multiuser Detection. Cambridge University Press, 1998.

[32] W. C. Jakes, Microwave Mobile Communications. John Wiley and Sons, 1974. 


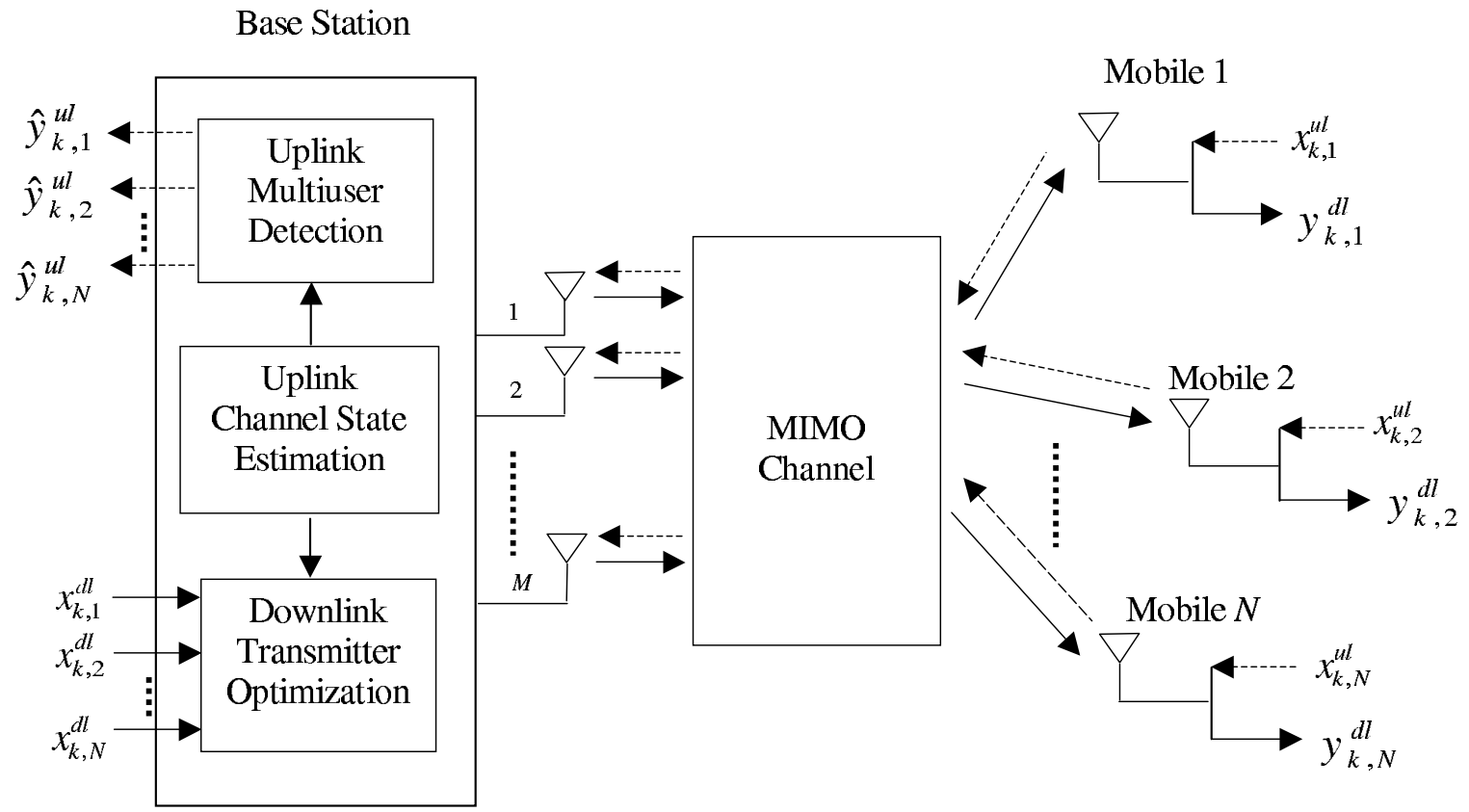

Figure 1: Multiple antenna multiuser TDD system, where $x_{k, n}^{u l}$ and $x_{k, n}^{d l}$ are signals transmitted on the uplink and downlink, respectively, $\hat{y}_{k, n}^{u l}$ is the uplink decision statistics and $y_{k, n}^{d l}$ is the downlink received signal, all corresponding to time instant $k$ and mobile terminal $n$.



Figure 2: Arrangement of the pilot and data-carrying symbols on the uplink, where $x_{k, n}^{u l}$ is the transmitted signal, $p_{k, n}^{u l}$ and $d_{k-N, n}^{u l}$ are the pilot and data symbols all corresponding to time instant $k$ and mobile terminal $n$. 


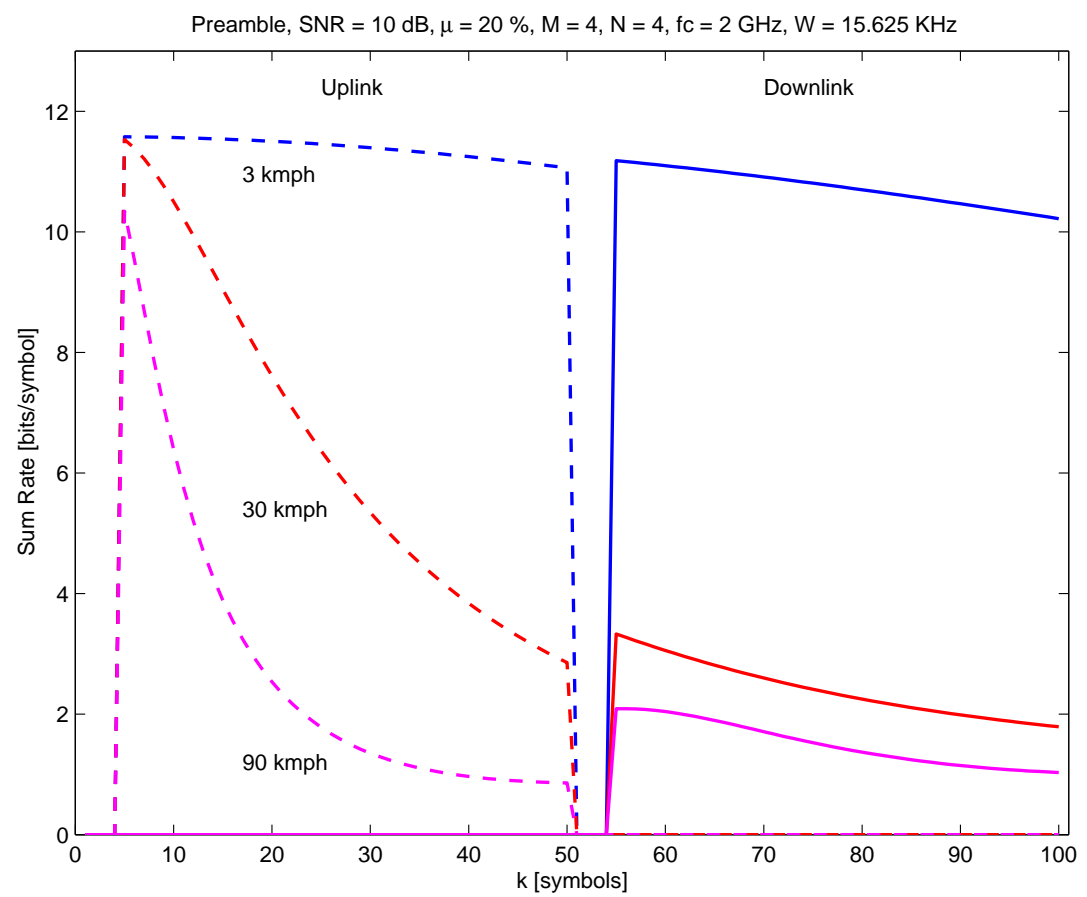

Figure 3: The sum of rates in (9) and (20) as a function of time, with the uplink pilot preamble.

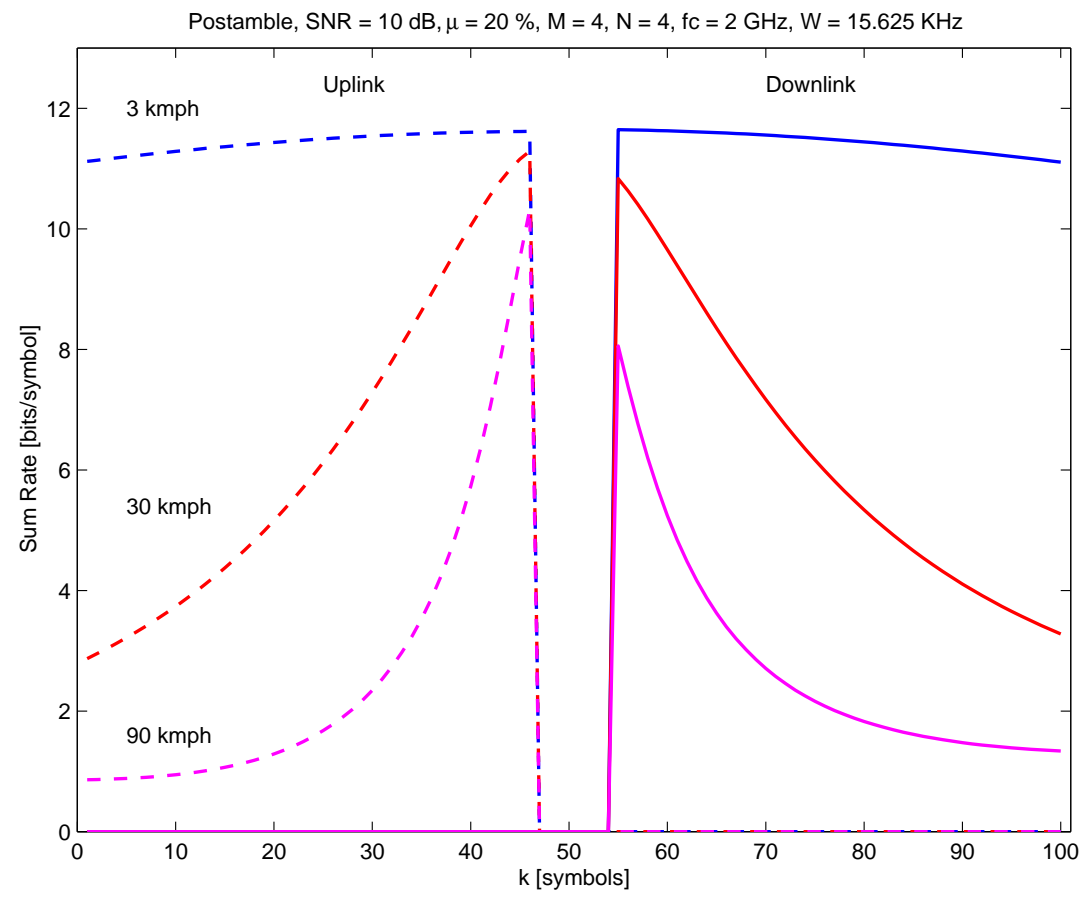

Figure 4: The sum of rates in (9) and (20) as a function of time, with the uplink pilot postamble. 


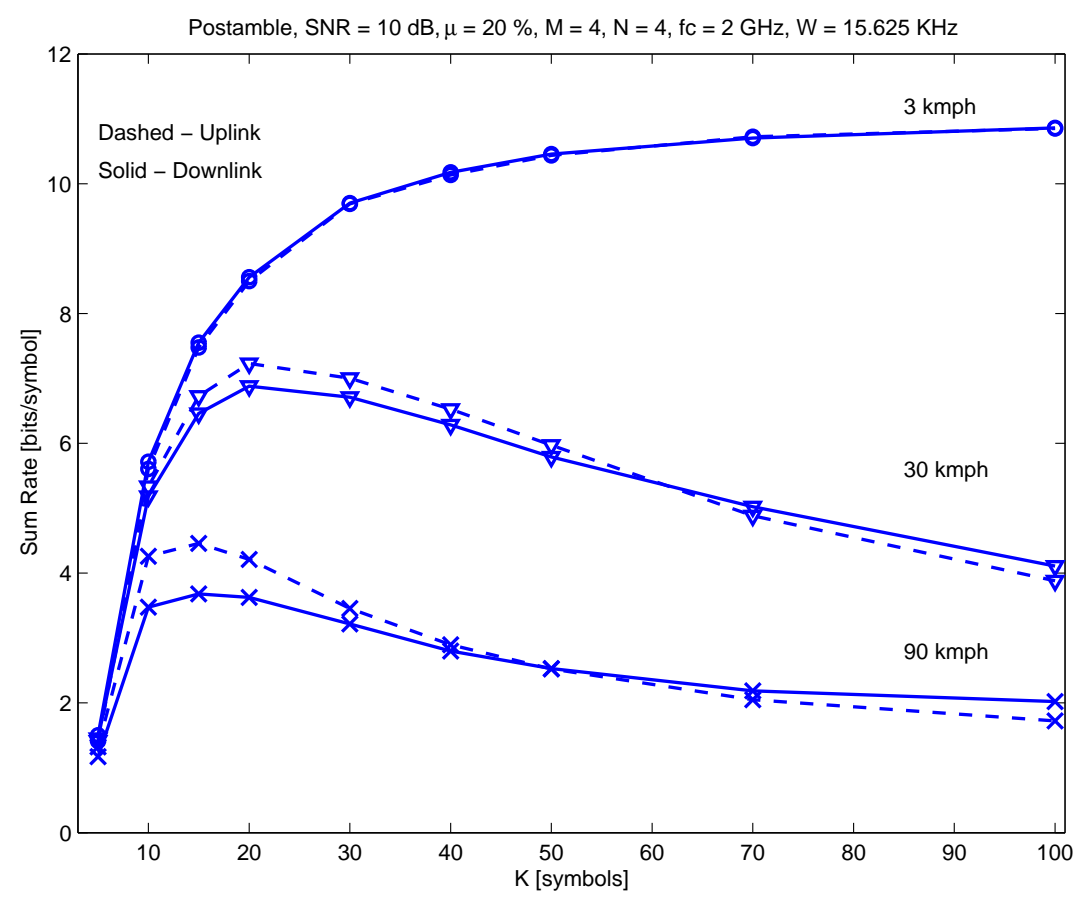

Figure 5: The sum of rates in (10) and (19) as a function of the uplink and downlink transmission interval.

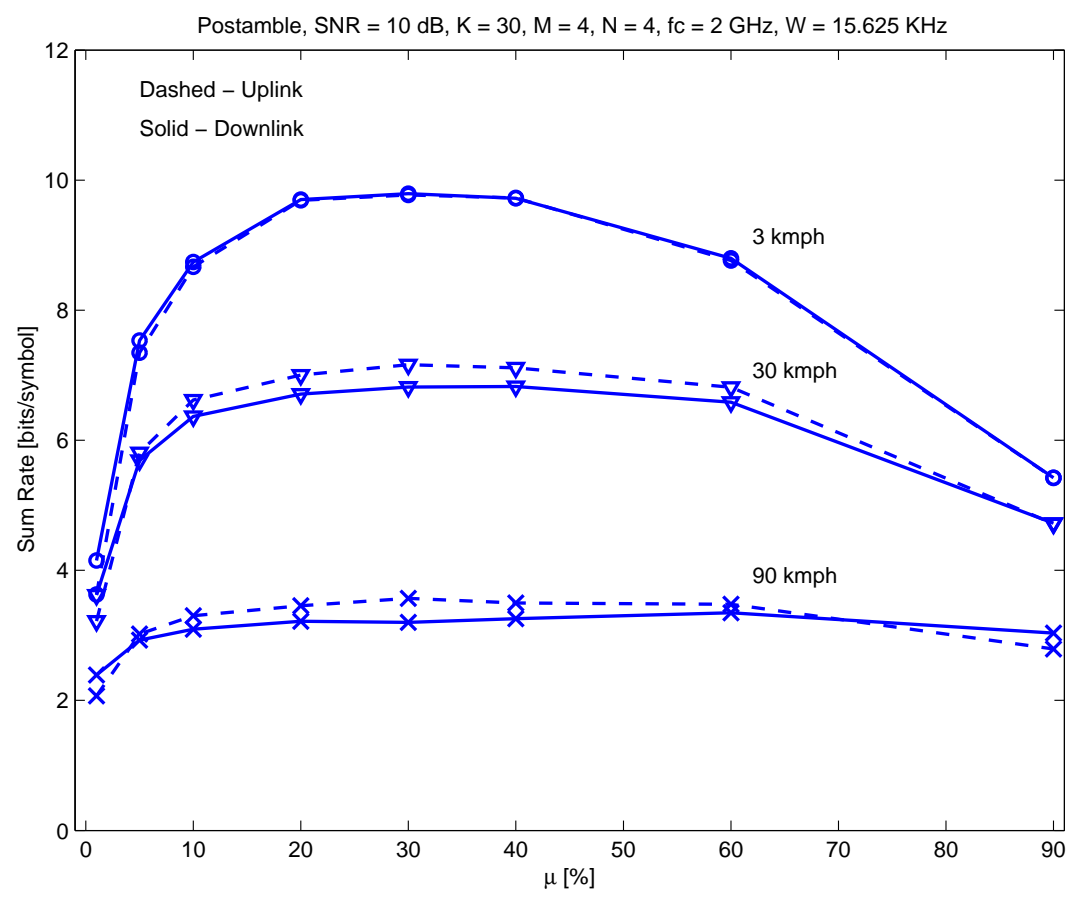

Figure 6: The sum of rates in (10) and (19) as a function of the percentage pilot power. 


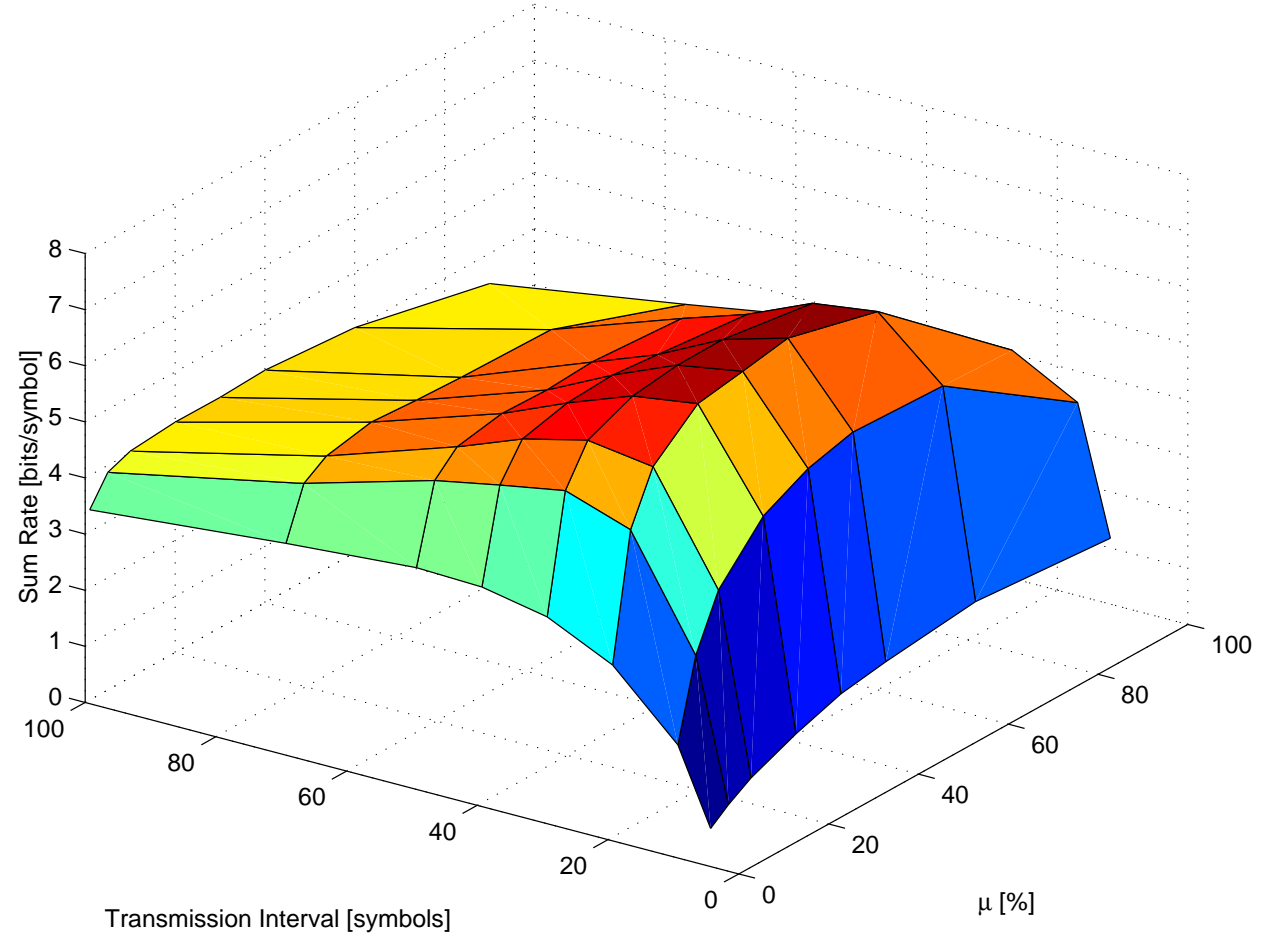

Figure 7: The sum of rates in (19) as a function of the transmission interval and the percentage pilot power.



Figure 8: The sum of rates in (10) and (19) as a function of $S N R$ and the mobile terminal speeds. 